\title{
Penile ossification: A traumatic event or evolutionary throwback? Case report and review of the literature
}

\author{
Ibrahim Edhem YIImaz, MD; ${ }^{*}$ Yagil Barazani, $M D ;{ }^{\dagger}$ Basir Tareen, $M D^{\S}$ \\ *Marmara University School of Medicine, Istanbul, Turkey; †Urology Resident, Beth Israel Medical Center, New York, NY; §Metropolitan Urologic Associates, Woodbury, MN
}

Cite as: Can Urol Assoc J 2013;7:E1 12-E1 14. hitp://dx.doi.org/10.5489/cuai.249

\section{Abstract}

Penile ossification is very rare, with only a handful of histologically confirmed reported cases. The most common condition leading to penile ossification is Peyronie's disease. Other conditions, such as gout, end-stage renal disease, diabetes mellitus, hyperparathyroidism and local trauma, have also been associated with penile ossification. We report a unique case of near-complete penile ossification of the corporal bodies with histologic confirmation on pathologic review. Our report summarizes the literature regarding this rare entity.

\section{Introduction}

Pathologic calcification or ossification refers to the process by which calcium salts build up in soft tissue, causing it to harden and form extraskeletal bone. This may result from a malignant infiltrative process, hypercalcemia secondary to a paraneoplastic syndrome, end stage renal disease, calciumphosphate abnormalities caused by hyperparathyroidism or other metabolic derangements, or from a local metaplastic process resulting from repeated trauma or a chronic inflammatory state.

Ossification occurring in the human penis is exceedingly rare, with fewer than 40 cases reported. Another related condition, "congenital human os penis," is also extremely rare, with only 1 reported case in a 5 -year-old boy. ${ }^{1}$ Ossification of the penis is most commonly due to Peyronie's disease, a chronic inflammation of tunica albuginea that leads to penile fibrosis. The hardened plaque reduces flexibility and leads to a penile bend or curvature during erection. Less common etiologies of penile ossification have been reported, including local trauma to the penis, chronic hemodialysis in patients with end-stage renal disease, chronic inflammatory states as in syphilis and gonorrhea, and with general metabolic disorders such as gout and diabetes.
We report a unique case of penile ossification of the corporal bodies with histologic confirmation.

\section{Case report}

A 54-year old gentleman presented to the urology office with a 1-year history of a painless hard proximal penile masses involving one-third the length of his corporal bodies bilaterally. He was neither sexually active nor bothered by symptoms from this penile lesion; he presented, however, for workup concerning the possibility of malignancy. The patient denied any history of trauma or family history of genitourinary malignancy. He also denied penile pain, dysuria, irritative voiding symptoms or any other subjective complaints. To the patient's knowledge, the hard mass had been present for several years and gradually increased in size over time.

Physical examination revealed a mobile, rock-hard, calcified mass palpable at the base of the penis circumferentially involving both proximal corpora. Digital rectal examination was normal and no inguinal nodes were palpable on examination. Results from routine laboratory evaluations were normal. A magnetic resonance imaging of the pelvis with gadolinium failed to identify any corporal abnormalities, and no pelvic lymphadenopathy was seen on imaging.

At this point, the decision was made to perform a cystoscopy and excisional biopsy of the calcified mass at the base of the penis. Cystoscopy revealed a normal urethra, bladder mucosa, and prostate, confirming that the plaque was external to the urethra. After degloving the phallus, the hard calcified proximal corpora were easily palpated and felt to be entirely replaced by a calcific process. An excisional biopsy was performed of the right corpora, with minimal bleeding noted from the calcified corporal body. Histological examination of this specimen revealed metaplastic ossification to lamellar bone with eosinophilic ossified matrix, lacunar spaces and haversian vascular canals characteristic of bone (Fig. 1). 


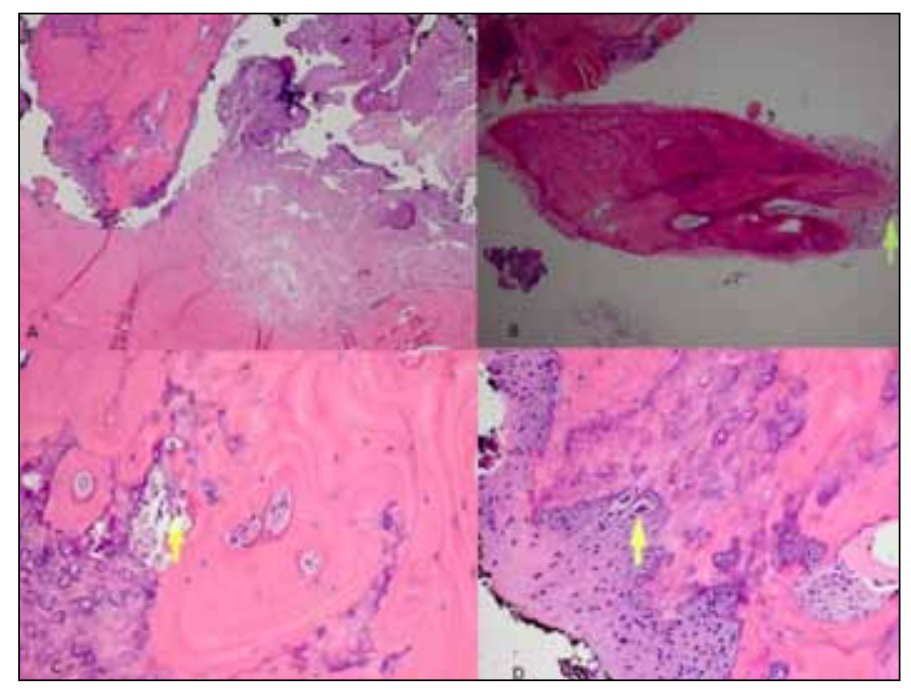

Fig. 1. A: Photomicrograph of histological section from the lesion, showing metaplasia of bone tissue in the corpus cavernosum. B: Osteoblastic rimming around bone tissue C: Osteoblasts. D: Multinucleated osteoclasts.

\section{Discussion}

The earliest case of penile ossification in the literature was reported by McClellan in 1827, ${ }^{2}$ who described a case of ossification along the full length of penis. In 1899 Chetwood reported a second case of ossification of corpora cavernosa in a postmortem specimen of a 55 -year-old diabetic man. ${ }^{3}$ In 1933, Vermooten described a 19-year-old male in whom a bony mass had developed in the glans penis. ${ }^{4}$ The patient also had a gunshot wound at that site 3 months previously. Presumably ossification had taken place in fibrosis resulting from this injury. A fourth case of penile ossification was reported by Eglitis in $1953 .{ }^{5}$ In this case, microscopic examination of sections taken from a grossly normal penis at autopsy identified bony plaques in the subcutaneous tissue, with no clues as to the etiology of this ossification. In 1962, Elliot and Fischerman reported a case of a 68-year-old man who presented with a $5-\mathrm{cm}$ bone in his corpus spongiosum which required surgical excision. ${ }^{5}$ The man was previously diagnosed with gout; however, the bone formation was thought to be unrelated. Subsequent to these rare cases of penile ossification reported in the early literature, other cases of localized penile ossification secondary to Peyronie's disease have been reported. ${ }^{6}$

As the condition is so rare, controversy remains as to the etiology of penile ossification. Some have pointed to a possible connection between human penile ossification and the normally occurring os penis in animals. It is known from the literature that several species of mammals have a bone in their penis, located in the septum or in the glans penis itself. ${ }^{5}$ These are called baculum or os priapi, and their presence enables a male of the species to mate for an extended period of time with a female in the absence of well-developed erectile tissue. Baculum vary in size, shape, and function from one species to the next. For example, in whales it measures up to 2 meters in length. In wolves and bears this structure is needed to achieve a rapid erectile state of the penis for copulation strategy, while in the dog it serves as a channel for the urethra. According to evolutionists, the os penis has progressively diminished in size over the years. For example, in apes it is insignificantly small in size, measuring only 10 to $20 \mathrm{~mm}$, and in chimpanzees, our closest ancestors, all that remains are small pieces of bone in the glans penis.

In humans, the os penis seems to have been acquired during the aging process because almost all of the cases were observed in 50- to 80-year-old men. But the atavistic theories concerning the human os penis in evolutionary terms were further supported by the aforementioned case described by Vermooten. ${ }^{4}$ Because the patient was young and the bony fragments were present on the glans, Vermooten believed that there could be a relation to the os penis seen in animals despite the fact that the ossification site had a history of a bullet wound. His conclusion bolstered the earlier view championed by Chetwood in 1899, who found fibrous hardenings in his patient's glans penis and had concluded this to be the result of an evolutionary process. ${ }^{3}$ Finally, Champion and Wegrzyn described a case of congenital os penis in 1964 in a 5-year-old boy who had bone in his penis making its curvature fixed from the birth. ${ }^{7}$ As with the earlier cases, this observation was thought to strengthen the atavistic viewpoint.

Despite the early theories of an evolutionary explanation for the few cases of human penile ossification, more contemporary authors have theorized that there is no relationship between the rare ossification seen in certain parts of the fibrous structures of the human penis and the os penis seen in animals. In 1924, Furuta rejected the atavistic thoughts because the bone tissue does not fit organically to the form of the penis, instead presenting as inhibitory factor for copulation especially during erection. ${ }^{8}$ Similarly, Bett argued that the os penis in man was unlikely to be a phylogenetic structure because of its associated barrier to copulation. ${ }^{8}$

Today, more recent explanations for the origin of the bone tissue attribute the ossification to a metaplastic process. As reported throughout the literature, the human body is able to form bone tissue or cartilage in places affected by pathological conditions when connective tissue is present. Bone tissue is known to originate even in places having nothing in common with the skeleton, including the mammary gland, salivary gland and the testes. Many pathologists accept that fibrous tissue has the ability to transform into a new tissue, including bone, in the unique cases of human penile ossification. ${ }^{9}$ 
Yilmaz et al.

\section{Conclusion}

The etiology of penile ossification remains controversial. In most cases the ossification process in the penis appears to be a metaplastic process as a result of local trauma or a late manifestation of Peyronie's disease. In our patient's case, the ossification was not attributable to any prior trauma or medical condition (systemic or local) and its cause therefore remains a mystery.

Competing interests: None declared.

This paper has been peer-reviewed.

\section{References}

1. Champion RH, Wegrzyn J. Congenital os penis. J Urol 1964;91:663-4.

2. McClellan G. Ossification of the septum of the corpora cavernosa of the penis. J Med \& Surg 1872;71:256.

3. Chetwood CH. Presentation of pathollogical specimen of ossified plaque of corpora cavernosa. J Cutan Gnitourinary Dis 1899;179-231.

4. Vermooten V. Metaplasia in the penis. The presence of bone, bone marrow and cartilage in the glans. New Engl J Med 1933;209:368. http://dx.doi.org/10.1056/NEJM193308242090802

5. Eglitis JA. Occurence of bone tissue in the human penis. J Urol 1953;70:749.

6. Vapnek J, Lue TF. Heterotopic bone formation in the corpus cavernosum: a complication of papaverineinduced priapism. J Urol 1989;142:1323-4.

7. Champion RH, Wegrzyn J. Congenital os penis. J Urol 1964;91:663-4.

8. Bett WR. The os penis in man and beast. Ann R Coll Surg Engl 1952;10:405-9.

9. Sarma DP, Weilbaecher TG. Human os penis. Urology 1990;35:349-50. http://dx.doi. org/10.1016/0090-4295(90)80163-H

Correspondence: Dr. Yagil Barazani, Urology Resident, Beth Israel Medical Center, Phillips Ambulatory Care Center, Suite 3A, 10 Union Square East, New York, NY 10003; ybarazani@chpnet.org 